\title{
Detection of Mycobacterium tuberculosis in Dog of Assam
}

\author{
Acheenta G. Barua ${ }^{1}$, Koushik Kakoty ${ }^{1}$, Pranjal M. Nath ${ }^{1}$ and Bhaben Baishya ${ }^{2}$ \\ ${ }^{1}$ Department of Veterinary Public Health, ${ }^{2}$ Teaching Veterinary Clinical Complex, College of \\ Veterinary Science, Assam Agricultural University, Khanapara, Guwahati-781022, India \\ *Corresponding author
}

\begin{abstract}
A B S T R A C T
Keywords

Dog,

Mycobacterium,

TST, IFN- $\gamma$ assay

Article Info

Accepted:

12April 2019

Available Online:

10 May 2019

A total of 52 suspected dogs with symptoms of harsh, chronic non-productive coughing, fever and anorexia were examined for tuberculosis in the Teaching Veterinary Clinical Complex, Guwahati, Assam. SIT carried out by injecting $0.1 \mathrm{ml}$ of tuberculin (2TU, 5TU and 10TU) at either the skin of the thorax at the level of the costochrondral junction or the skin on the medial aspect of the hind limb in the thigh region and blood was collected aseptically for IFN- $\gamma$ assay. Out of which the 2 dogs were found positive for TST and 3 dogs were positive for IFN-gamma assay. Moreover, X-ray was carried out in 4 suspected dogs and only one dog had shown suspected lesion in the lung. Post-mortem was also carried out in 3 suspected tuberculosis cases brought to the Teaching Veterinary Clinical Complex (TVCC), Guwahati, Assam. An isolate of Mycobacterium was recovered from the suspected lesion test and confirmed as Mycobacterium tuberculosis by biochemical test and PCR assay. These parameters may be used for diagnosis of tuberculosis in dog.
\end{abstract}

\section{Introduction}

Natural infection by Mycobacterium tuberculosisis uncommonly isolated from cases of animal tuberculosis following close, prolonged contact with infectious humans (Michel et al., 2003). Companion animals living in contact with TB patients are at great risk of exposure to this pathogen and post mortem surveys performed in European cities in the first half of the 20th century determined the prevalence of canine $\mathrm{TB}$ as varying between 0.1 and $6.7 \%$ (Snider, 1971). Also the diseased companion animals can be the potential source of infection to human has been highlighted by previous author (Snider 1971).

Tuberculosis is one of India's major public health problems. According to WHO estimates, India has the world's largest tuberculosis epidemic (WHO, 2006). In such environments, epidemiological investigations of both non-clinical M. tuberculosis infection and clinical TB disease have evidenced high levels of $M$. tuberculosis transmission between people and it can be expected that companion animals living in such environments will be at particular risk of infection by this pathogen. However, TB is 
prevalent in resource-poor settings in which sophisticated veterinary services are generally unavailable and where cases of canine TB will remain largely undetected.

In addition to the lack of clinical data about canine TB, a comprehensive understanding of this disease is further limited by the absence of practical immunological tests for the diagnosis of both clinical disease and nonclinical $M$. tuberculosis infection in this species. These tests typically rely on the detection of antigen-specific $\mathrm{T}$ lymphocytemediated responses as surrogate markers of infection by the causative organism (de la Rua-Domenech et al., 2006). This principle is employed in the in vivo tuberculin skin test (TST) which characterises the inflammatory response to mycobacterial purified protein derivative (PPD). Similarly, the more recently described interferon-gamma (IFN-c) release assays (IGRA) quantify the in vitro release of IFN-c by lymphocytes stimulated by $M$. tuberculosis-specific antigens. Currently, no standard protocols exist for the TST in canines as it has long been believed that this test is unreliable in dogs and that the use of regular M. tuberculosis and M. bovis PPD as TST stimuli are uninformative (Bonovska et al., 2005).

The present study was undertaken for the purpose to investigate the occurrence of $M$. tuberculosis infection in dogs by TST, IFN- $\gamma$ and PCR assay and to determine the risk of transmission of $M$. tuberculosis from infectious human TB patients to contact dogs.

\section{Materials and Methods}

Fifty two dogs with symptoms of harsh, chronic non-productive coughing, fever and anorexia were brought to the Teaching Veterinary Clinical Complex, Guwahati, considering as a referral centre for disease diagnosis and treatment.

\section{Single TST}

Prior to the TST, dogs were sedated with Thiopental Sodium (25 mg/kg, i/v) (Thiosol 1gm, Neon Laboratories Ltd., Mumbai, India). The TST was done by 3 intradermal injections of $0.1 \mathrm{ml}$ of 2TU, 5TU and 10TU of Tuberculin PPD (Arkray Healthcare Ltd., Gujrat, India) in the medial aspect of thigh. Skin thicknesses were measured at both sites before the intradermal injection and after 72 hrs. If the skin thickness is more than $5 \mathrm{~mm}$, it is considered as positive (OIE).

\section{IFN- $\gamma$ assay}

Blood samples were collected aseptically for IFN- $\gamma$ assay. It was performed according to kit procedures (life technologies IFN- $\gamma$ canine ELISA kit). Samples were read at a wavelength of $450 \mathrm{~nm}$ to calculate optical density. A sample was considered as positive when the difference between mean optical density value of a negative control with mean optical density value of sample is equal or higher than 0.100 .

\section{Radiographic studies}

Chest radiography was made of each animal in the dorso-ventral and left recumbent position to determined opaque image in lung lobes, military lesion in the lungs and heart, enlargement of the liver, spleen, hilar and mesenteric lymph node.

\section{Gross necropsy}

Carcasses were inspected with the standard procedure for any gross visible lesion suspected of tuberculosis. Organs and tissue samples were collected from all the carcasses for further analysis. In this study, an animal was considered positive on necropsy if 1 or more lymph nodes or other tissues contained focal or multifocal abscesses or granulomas. 
Mycobacterial culture and species identification

Fresh samples were macerated and decontaminated using NALC and inoculated on to Lowenstein Jensen (LJ) media. Briefly, approximately $1 \mathrm{~g}$ of tissue exhibiting gross visible lesions was sliced and homogenized and then subjected for decontamination.

The supernatant was discarded and the pellet formed re-suspended in $300 \mu \mathrm{l}$ of phosphate buffered saline $(140 \mathrm{mMNaCl}, 26 \mathrm{mM} \mathrm{KCl}$, $10.0 \mathrm{mM}$ Na2HPO4 and $1.7 \mathrm{mM}$ KH2PO4). Then the re-suspended pellets were inoculated in duplicates onto LJ slants (one incorporating glycerol and the other pyruvate). LJ slants were incubated at $37 \mathrm{oC}$ and observed weekly for eight weeks. Using a sterile $0.1 \mu \mathrm{l}$ plastic loop, the re-suspended pellets were spread and fixed at $80 \mathrm{oC}$ (for $10 \mathrm{~min}$ ) onto a labelled slide. The slides were subjected for staining with modified ZN stain.

Biochemical analysis were performed for species identification of mycobacteria as per standard protocol, such as Nitrate reduction test (Kubica and Wayne, 1984), Pyrazinamidase test (Wayne, 1974) and Niacin detection test (Gadre et al., 1995).
DNA was isolated from bacterial culture and PCR was done targeting hsp65 gene amplifying $441 \mathrm{bp}$ as per De Los Monteros et al., (1998).

\section{Results and Discussion}

In the current study, we assayed dogs with TST and IFN- $\gamma$ and necropsy tissue samples with lesions suggestive of mycobacterial infection from post-mortem dog using $\mathrm{ZN}$ microscopy and compared the results with those of culture, biochemical tests and PCR. A total of 52 suspected dogs were tested by using the TST and IFN- $\gamma$ assay and out of which the 2 dogs were found positive for TST (Fig. 1) and 3 dogs were positive for IFN- $\gamma$. Although precise determination of sensitivity and specificity of each of the PPD employed is not possible, it would be appear that TST results were inconsistent with those of the IFN- $\gamma$. The IFN- $\gamma$ assay is advantageous over the TST because IFN- $\gamma$ assay has been designed to be highly specific by using welldefined antigens, and it allows for the inclusion of positive and negative controls. Together, these findings support those of other studies which have found the TST ineffective in dogs (Bonovska et al., 2005).

Fig.1 A) $0.1 \mathrm{ml}$ PPD (2, 5 and $10 \mathrm{TU})$ injected intradermally in the shaved area ofmedial aspect of the hind limb in the thigh region. B) Positive results after $48 \mathrm{hrs}$ for $5 \mathrm{TU} .(<5 \mathrm{~mm})$

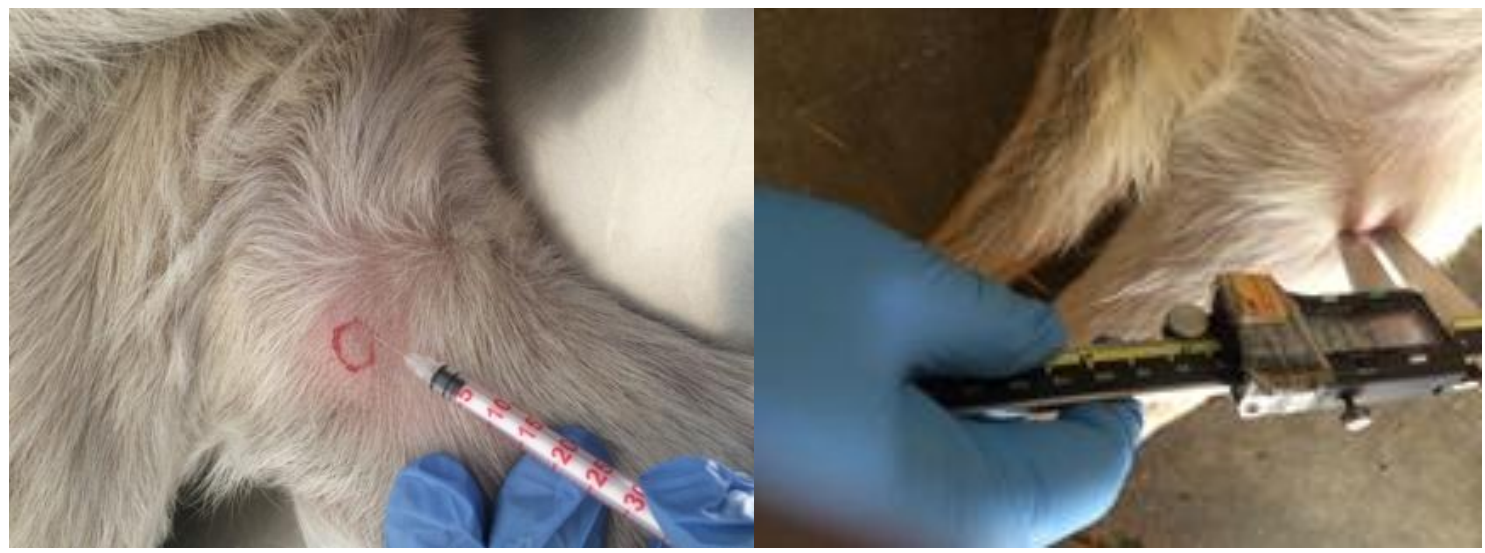

A

B 
Fig.2 Survey radiography of the thorax. A) Suspected lesion in the right lobe of lung (arrow) B) Pleural effusion can been seen (SIT positive reactor)

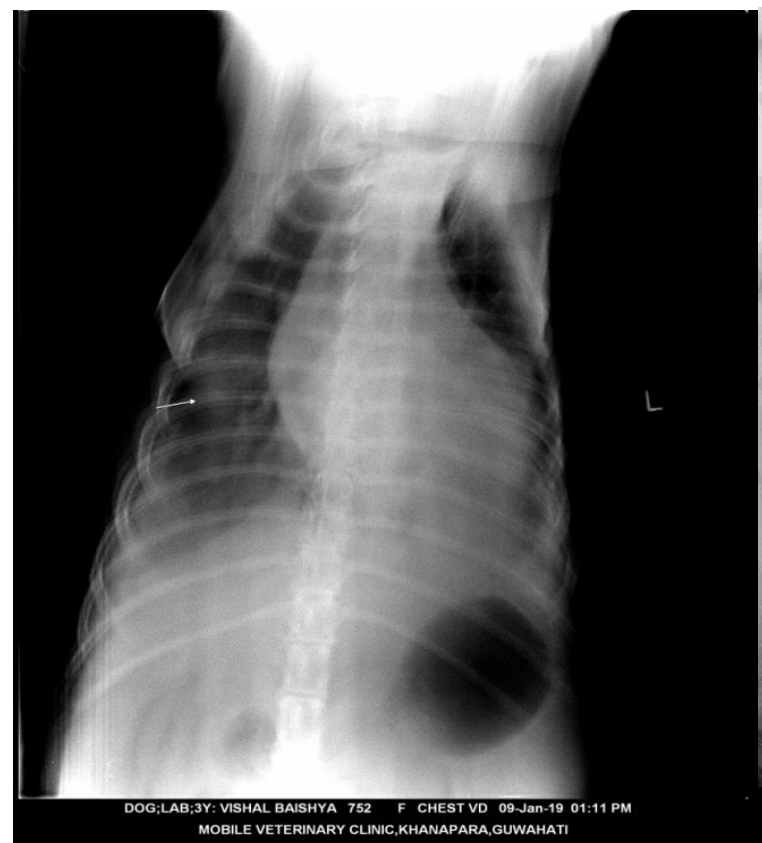

A

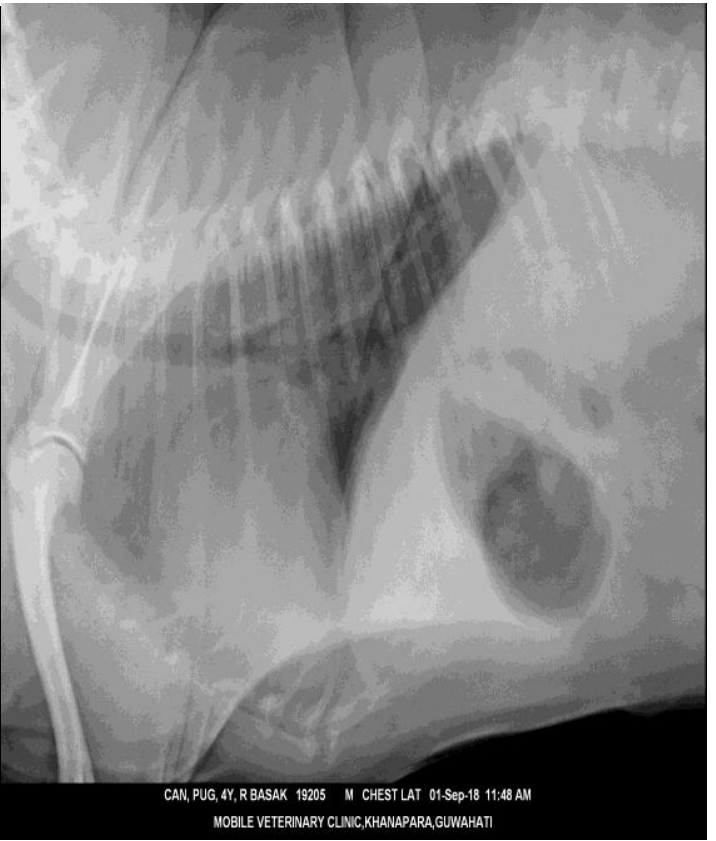

B

Fig.3 Areas of liquifactive necrosis, on incision creamy white materials ooze out

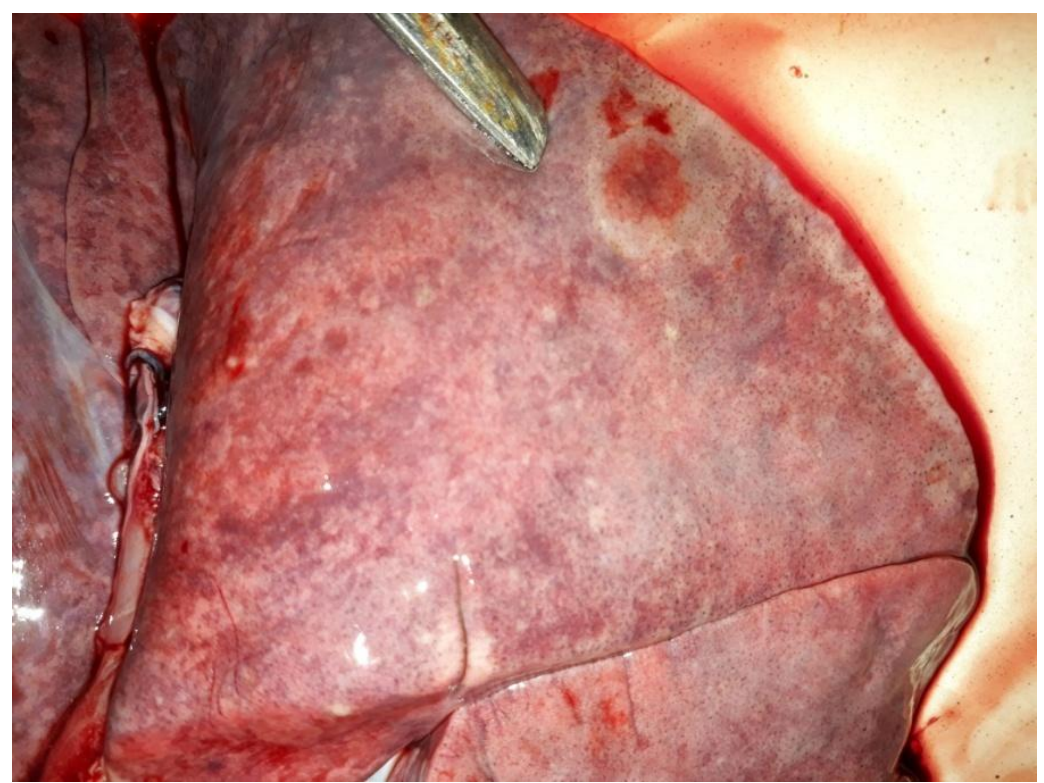


Fig.4 PCR assay of hsp65 gene for Mycobacterium tuberculosis complex; L: 100 bp ladder, M1\&M2: Isolates in duplicate, M3: Positive control and N: Negative control

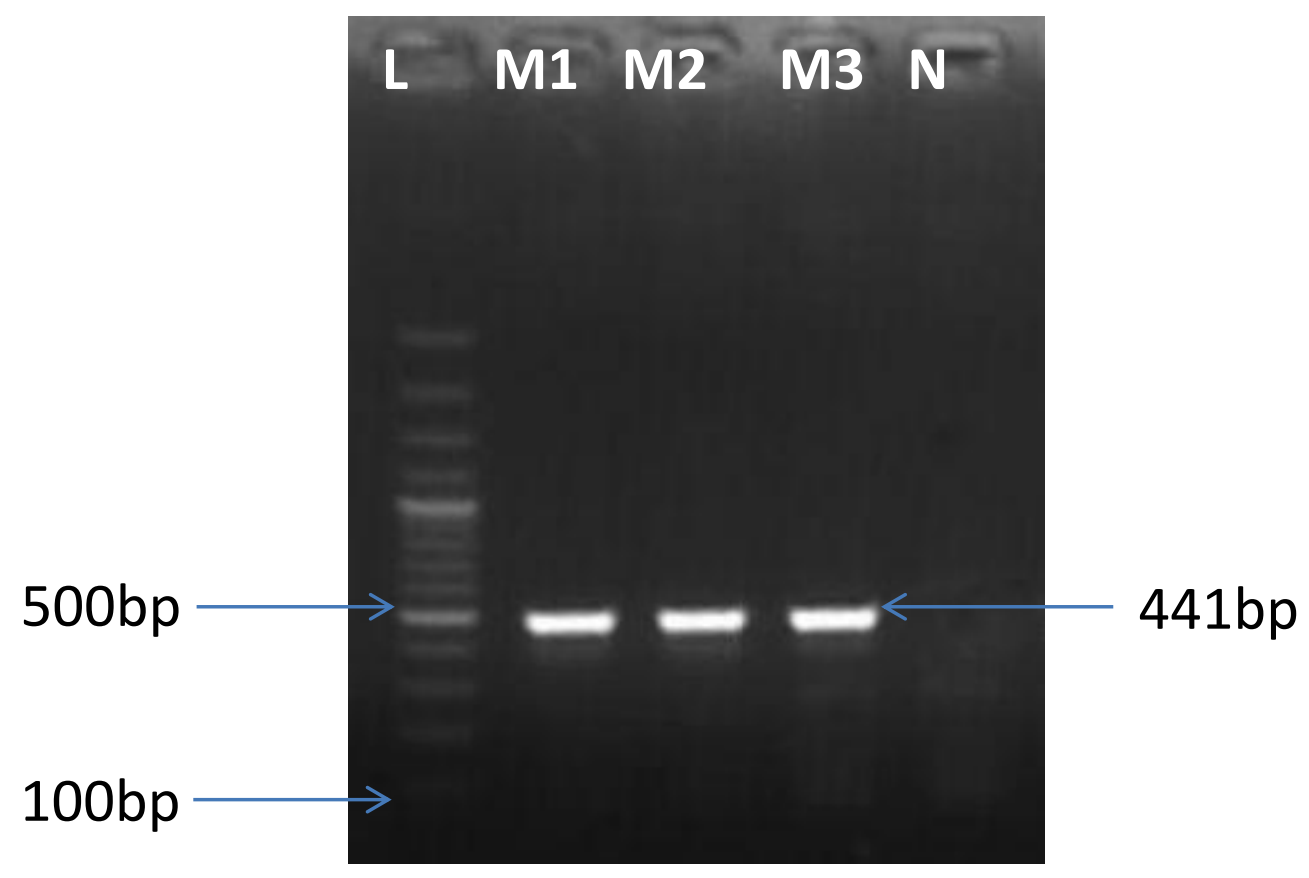

Moreover, X-ray was carried out in 4 suspected animals to detect pleural and pericardial effusion, ascites and hepatomegaly, diffuse radio-opaque images in lung lobes, diffuse visible masses in abdominal organs, hilar and mesenteric lymphadenopathy etc. However only one dog had shown suspected lesion in the lung (Fig. 2).

Post-mortem was also carried out in 3 suspected tuberculosis cases brought to the Teaching Veterinary Clinical Complex (TVCC), Guwahati, Assam. Out of which one dog was showing liquifactive necrosis on liver and the tissue sample was processed for isolation of Acid-fast ( $\mathrm{ZN}$ positively-stained) tuberculus bacteria. The isolate was recovered from the suspected lesion and confirmed as M. tuberculosis by biochemical test viz. Niacin production, nitrate reduction, urease production and PCR assay (Fig. $3 \& 4$ ). These results were well supported by Parsons et al., (2008). Though transmission of tuberculosis between human and $\operatorname{dog}$ is not well established, pet owner, veterinarian, physicians and public should be aware of the potential transmission. However, culture and molecular assay will be helpful in understanding the dynamics of tuberculosis between human and dog.

\section{Acknowledgement}

Authors are thankful to Department of Biotechnology for funding the "Molecular Epidemiology of Canine Tuberculosis in Assam, neighboring States and its Containments" and Director of Research (Veterinary) for necessary facilities to carry out the research. Due acknowledgement is also extended to the abattoir workers for providing samples.

\section{References}

Bonovska, M., Tzvetkov, Y., Najdenski, H. and Bachvarova, Y. 2005. PCR for 
detection of PCR for detection of Mycobacterium tuberculosis in experimentally infected dogs. Journal of Veterinary Medicine. B, Infectious Diseases and Veterinary Public Health, 52: $165-170$.

de la Rua-Domenech, R., Goodchild, A.T., Vordermeier, H.M., Hewinson, R.G., Christiansen, K.H., Clifton-Hadley, R.S., 2006. Ante mortem diagnosis of tuberculosis in cattle: a review of the tuberculin tests, c-interferon assay and other ancillary diagnostic techniques. Research in Veterinary Science 81, 190-210.

de los Monteros L.E.E., Galán, J.C., Montserrat Gutiérrez, Samper, S., Marín, Carlos Martín, J.F.G., Domínguez, L., de Rafael, L., Baquero, F., Enrique Gómez-Mampaso, E.G. and Blázquez, J., 1998. Allele-specific PCR method based on pncA and oxyR sequences for distinguishing Mycobacterium bovis from Mycobacterium tuberculosis: intraspecific $M$. bovis pncA sequence polymorphism. J. Clin. Microbiol., 36: 239-242.

Gadre, D. V., Mahajan, M., Singh, N. R., Agarwal, D. S. and Talwar, V. 1995. Niacin test for mycobacteria: a comparative study of two methods. Ind. J. Tub., 42: 225-226.
Kubica, G. P and Wayne, L. G. 1984.Clinical microbiology. In: The Mycobacteria, a source book, Part A. Marul Dekker, Inc, New York, Basel, pp. 156.

Michel, A.L., Venter, L., Espie, I.W. and Coetzee, M.L. 2003. Mycobacterium tuberculosis infections in eight species at the National Zoological Gardens of South Africa, 1991-2001. Journal of Zoo and Wildlife Medicine, 34: 364370.

OIE terrestrial manual, Bovine tuberculosis, Chapter 2.4.6, http://www.oie.int/en/ international-standard-setting/terrestrialmanual/access-online.

Parsons, S.D.C., Gous, T.A., Warren, R.M. and van Helden, P.D., 2008. Pulmonary Mycobacterium tuberculosis (Beijing strain) infection in a stray dog. Journal of the South African Veterinary Association, 79: 95-98.

Snider, W.R., 1971. Tuberculosis in canine and feline populations. American Review of Respiratory Disease, 104: 877-887.

Wayne, L. G. 1974. Simple pyrazinamidase and urease test for routine identification of Mycobacteris. Am. Rev. Resp. Dis., 109: 147-51.

WHO. Global tuberculosis control. WHO report. WHO/HTM/TB/2006.362. Geneva: World Health Organization, 2006.

\section{How to cite this article:}

Acheenta G. Barua, Koushik Kakoty, Pranjal M. Nath and Bhaben Baishya. 2019. Detection of Mycobacterium tuberculosis in Dog of Assam. Int.J.Curr.Microbiol.App.Sci. 8(05): 1283-1288. doi: https://doi.org/10.20546/ijcmas.2019.805.146 